\title{
Implementation of Body Area Networks Based on MICS/WMTS Medical Bands for Healthcare Systems
}

\author{
Mehmet R. Yuce and Chee Keong Ho \\ The School of Electrical Eng. and Computer Sci., University of Newcastle, Callaghan, NSW 2308, Australia. \\ e-mail: mehmet.yuce@newcastle.edu.au
}

\begin{abstract}
A multi-hoping sensor network system has been implemented to monitor physiological parameters from multiple patient bodies by means of medical communication standards MICS (Medical Implant Communication Service) and WMTS (Wireless Medical Telemetry Service). Unlike the other medical sensor networks (they usually use $2.4 \mathrm{GHz}$ ISM band), we used the two medical standards occupying the frequency bands that are mainly assigned to medical applications. The prototype system uses the MICS band (402-405 MHz) between the sensor nodes and a remote central control unit (CCU). And WMTS frequencies $(608-614 \mathrm{MHz})$ are used between the CCUs and the remote base stations allowing for a much larger range acting as an intermediate node. The sensor nodes in the prototype can measure up to four body signals (i.e. 4-channel) where one is dedicated to a continuous physiological signal such as ECC/EEG. The system includes firmware and software designs that can provide a long distance data transfer through the internet or a mobile network.
\end{abstract}

\section{INTRODUCTION}

One of the many applications of Wireless Body Area Networks (WBAN) is in medical environment where conditions of patients are continuously monitored in real time. Wireless monitoring of physiological signals from a large number of patients is one of the current needs in order to deploy a complete wireless sensor network in healthcare systems. Such an application presents some challenges in both software and hardware designs. Some of them as follows: reliable communication by eliminating collisions of two patients' signals and interference from other external wireless devices, low-cost, low power consumption, and providing flexibility to the patients so that they can be relocated anytime. We have developed a multi-hopping wireless medical network prototype system that use MICS (Medical Implant Communication Service) [1][2] to obtain data from sensors placed on or in the body, and WMTS (Wireless Medical Telemetry Service)[3] as an intermediate node for a longer wireless communication range. Specifications of our devices with comparison to other proposed medical networks are summarized in Table-I.

Majority of the existing wireless systems for medical monitoring use standards such as ZigBee (IEEE 802.15.4), WLANs, and Bluetooth (IEEE 802.15.1)[4]-[10]. The current trend indicates that Bluetooth and WLAN technologies are avoided for low power sensor nodes because of their large size and power consumption used to provide longer ranges (i.e. $100 \mathrm{~m}$ ). On the other hand, these technologies can be useful for a wireless link between the control unit and a mobile device (i.e. PALM) or from the control unit to internet via a Wi-Fi link.

Low power operation is essential for sensor nodes as it determines the life time and the size of the device. Power consumption of sensor nodes is dominated by the operation of the wireless chip, RF transmission and reception. Thus it is desirable to use a wireless standard (or a wireless chip) that will provide low power consumption and has a minimum transmitted power while still meeting the required range of a body area network. The low rate IEEE 802.15.4 technology has been the most popular among all the short-range standards for medical monitoring due to its low transmitter power [11][12]. It may however suffer from the strong interference by WLANs because WLANs use the same multiaccess and transmit bigger signal power [13]. Installing an interference free medical network in a hospital may thus be quite challenging since there exist a lot of other wireless systems and equipments using the $2.4 \mathrm{GHz}$ band. The WBAN technologies operating at $2.4 \mathrm{GHz}$ ISM band should thus deal with the interference issues when they are located in the same environment [14] [15].

Table I. Comparison of technologies for WBAN

\begin{tabular}{|c|c|c|c|c|c|c|}
\hline & MICS & WMTS & $\begin{array}{c}\text { UWB IEEE } \\
(802.15 .6)\end{array}$ & $\begin{array}{c}\text { IEEE 802.15.4 } \\
\text { (Zigbee) }\end{array}$ & $\begin{array}{c}\text { IEEE 15.1 } \\
\text { (Bluetooth) }\end{array}$ & $\begin{array}{c}\text { WLANs } \\
(802.11 b / g)\end{array}$ \\
\hline Frequency bands & $402-405 \mathrm{MHz}$ & $\begin{array}{c}608-614 \mathrm{MHz}, \\
1395-1400 \mathrm{MHz}, \\
1429-1432 \mathrm{MHz}\end{array}$ & $3-10 \mathrm{GHz}$ & $\begin{array}{c}2.4 \mathrm{GHz} \\
\text { (868/915MHz } \\
\text { Eur./US) }\end{array}$ & $2.4 \mathrm{GHz}$ & $2.4 \mathrm{GHz}$ \\
\hline Bandwidth & $3 \mathrm{MHz}$ & $6 \mathrm{MHz}$ & $>500 \mathrm{MHz}$ & $5 \mathrm{MHz}$ & $1 \mathrm{MHz}$ & $20 \mathrm{MHz}$ \\
\hline Data rate & 16 kbps (AMIS)* & 76 kbps* & $850 \mathrm{kbps}$ & $250 \mathrm{kbps}(2.4 \mathrm{GHz})$ & $1 \mathrm{Mbps}$ & $>11 \mathrm{Mbps}$ \\
\hline "Multiple Access & $\begin{array}{l}\text { CSMA/CA, } \\
\text { Polling }\end{array}$ & $\begin{array}{l}\text { CSMA/CA, } \\
\text { Polling }\end{array}$ & ALOHA & CSMA/CA & FHSS & $\begin{array}{c}\text { OFDMA, } \\
\text { CSMA/CA }\end{array}$ \\
\hline Tx Power & $-16 \mathrm{dBm}(25 \mu \mathrm{W})$ & $\begin{array}{l}\geq 10 \mathrm{dBm} \text { and }< \\
1.8 \mathrm{~dB} \text { (1.5 watt) }\end{array}$ & $-41 \mathrm{dBm}$ & $0 \mathrm{dBm}$ & $4 \mathrm{dBm}, 20 \mathrm{dBm}$ & $250 \mathrm{~mW}$ \\
\hline Range ** & $0-10 \mathrm{~m}$ & $>100 \mathrm{~m}$ & $2 \mathrm{~m}$ & $0-10 \mathrm{~m}$ & $10,100 \mathrm{~m}$ & $0-100 \mathrm{~m}$ \\
\hline
\end{tabular}

\footnotetext{
* Note that MICS/WMTS bands can use a data rate more than $250 \mathrm{kbps}$. The indicated rates are specifications of the wireless chips used in our devices.
} **Transmission range for a medical sensor network has commonly been $10 \mathrm{~m}$ which will give enough space for a patient in a hospital room. 


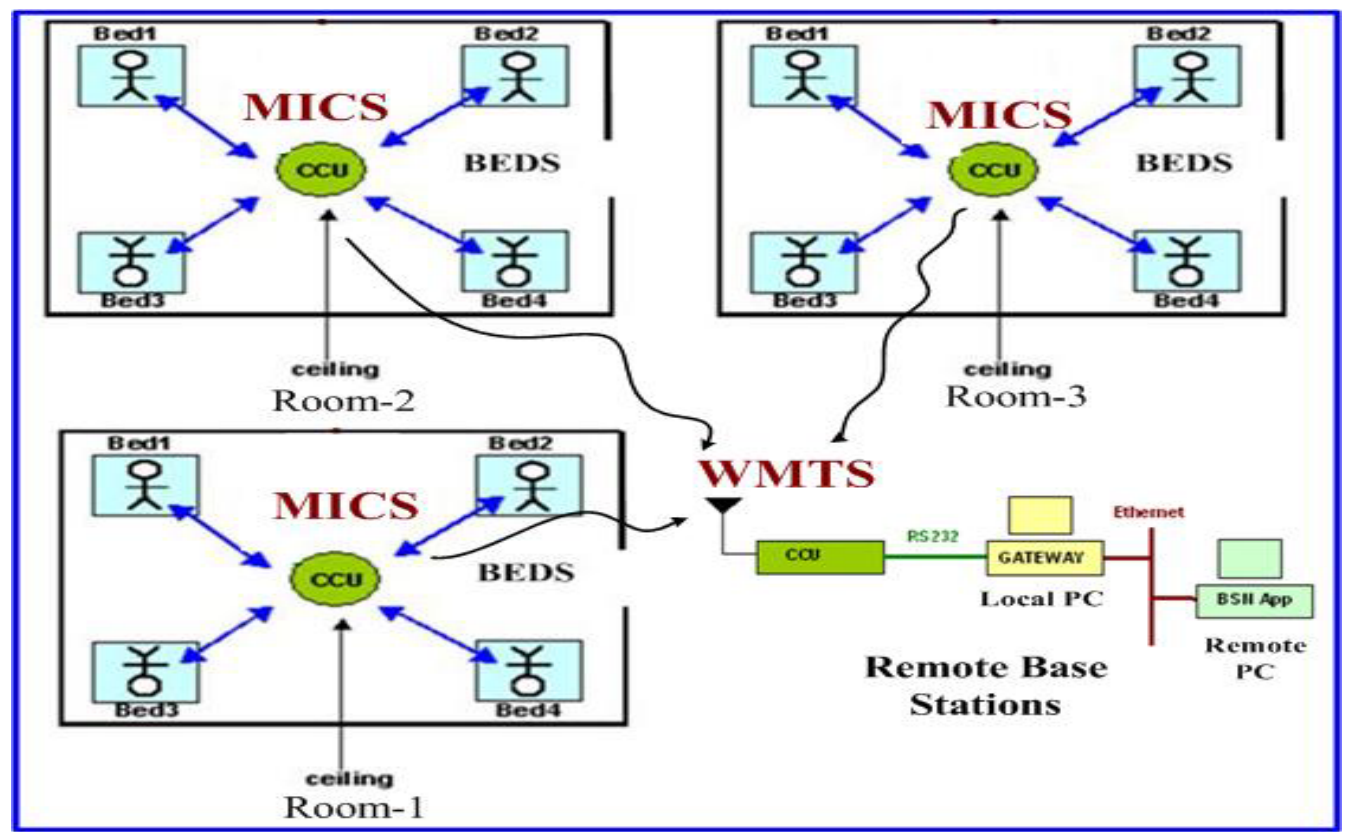

Figure-1. A wireless body area network system for multi-patient monitoring in medical centers.

A study group of IEEE has been launched in November 2007 to work on the WBAN standardization [16]. UWB has been considered as the potential wireless technology [17]. One big advantage of UWB wireless technology is its data rate range from $850 \mathrm{kbps}$ to $20 \mathrm{Mbps}$ which can be used for simultaneous monitoring of many continuous physiological signals such as ECC/EEG. Although the standard is still accepting proposals for the development of the body area networks, the UWB wireless chips are not available commercially to apply WBAN at the moment. Although UWB was claimed very low power initially in the literature, the attempts of such technology in the integrated circuits have exhibited power consumption more than that of the conventional narrowband short range wireless chips [18]. Since UWB operates at very high frequencies $(3-10 \mathrm{GHz}$ for medical applications), it has very high penetration loss which will significantly affect the performance and size of the implantable nodes in a WBAN. Thus a WBAN standard will most likely incorporate a narrow band together with the UWB technology to cover many environments in future.

In our multi-hopping structure, the MICS band is used for gathering signals from sensors and WMTS is used to provide a longer range monitoring. Both frequency bands are internationally available and are permitted for a remote monitoring of several patients simultaneously. The MICS band has low emission power ( $25 \mu \mathrm{W}$, comparable to UWB) and lower power consumption, and will thus provide one of the most suitable medical sensor nodes [20]. Although there have been few incidents reported due to the interference from some local TV channels in USA, WMTS is still the most popular band for wireless telemetry used in hospitals [21].

\section{Multi-Patient Monitoring via Body Area Network}

Hardware electronics and software programs are developed for the scenario shown in Fig. 1 in the proposed WBAN. Our initial WBAN was based on a wireless link between patient's sensors to CCU [19]. In this figure, we cover a large environment that may represent a floor in a medical center. The center may have few rooms and each room may accommodate more than one patient. As depicted in Fig. 1, after obtaining raw data from a human body, sensor nodes transmit it to the central control unit (CCU) via the wireless RF link using the MICS band. The CCU then re-packages the data and transmits to the base station. The data is collected at a local PC which is transferred to a remote PC across the network in a medical center or through internet if it is at a location different than the medical center.

A CCU can be used for one individual in the medical center or can be used privately in a patient house. If more than one patient shares a CCU box. The CCU can either be connected to a local PC in the room or it can transmit data to another remote CCU box that is attached to a so called local PC via another wireless link using WMTS (600 MHz). We are using a multi-hopping network technique that consists of three networking structures in the system. A network between sensors CCU, another network between CCUs-base station, and the last communication is between base stations in the medical centers.

For multi-access communication protocol, we are using a combination of CSMA/CA and polling architecture [19]. The CSMA/CA MAC protocol is used by the IEEE802.11 based WiFi and the IEEE802.15.4 based WPAN (Wireless Personal Area Network) standards. This MAC protocol is most likely to be adopted by the IEEE802.15.6 WBAN (Wireless Body Area Network) standard [16] [17]. The CSMA/CA protocol is a contention based protocol which could offer lower delay and reliable transmission of packets in small size networks like a wireless body area network.

Fig. 2 shows our hardware setup for the multi-hoping communications. Patients' physiological parameters are sent to intermediate CCUs and then to the base station. MICS regulations require that the power output of any terminal must be kept under $-16 \mathrm{dBm}$ and is not intended for long range 


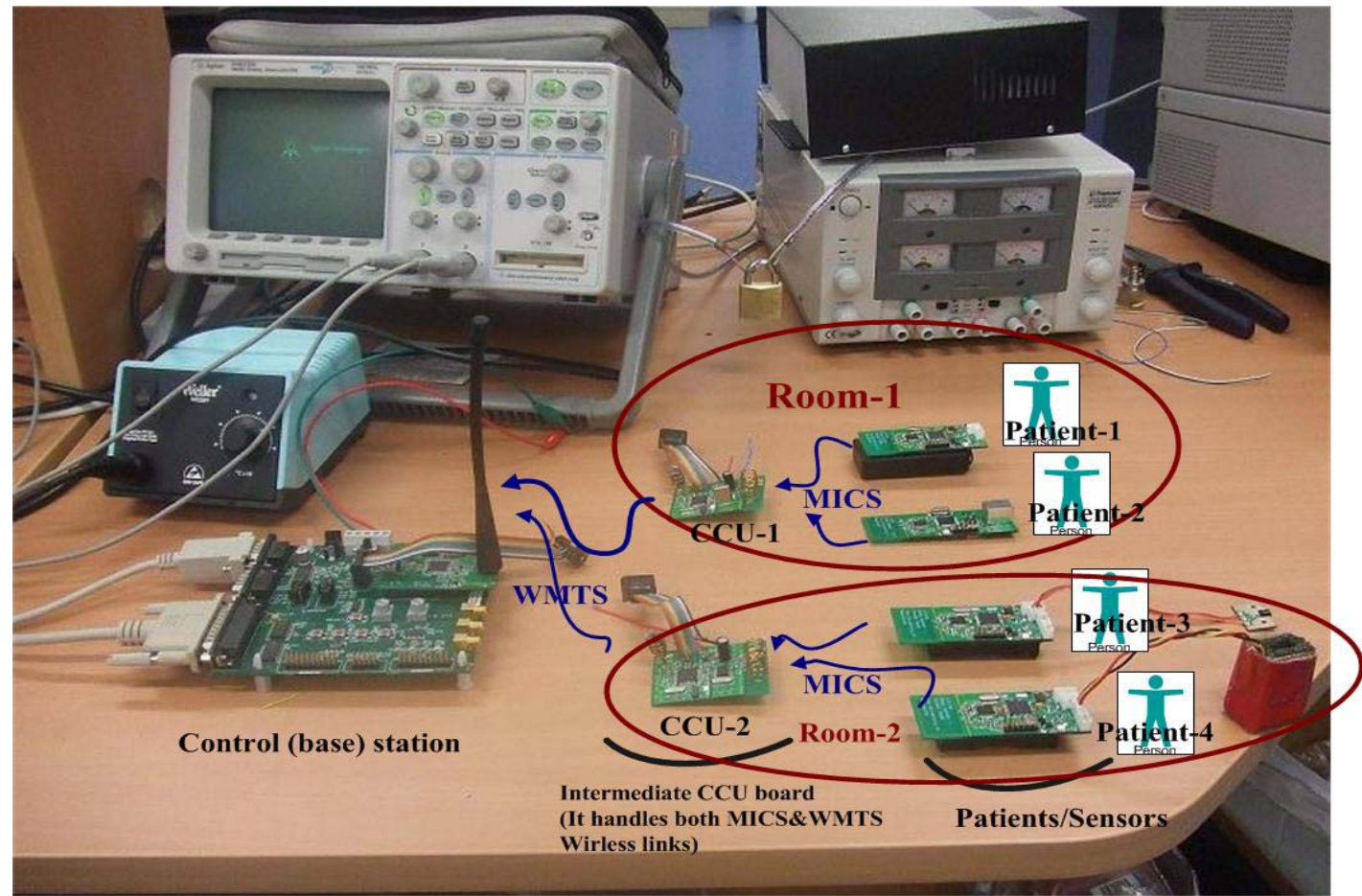

Figure. 2. A prototyping system for a multi patient monitoring: each sensor nodes have four channel serological signal detection capabilities and represents one patient. The prototyping system uses two medical standards MICS for short distance and WMTS for long distance wireless transmission respectively.

connections. To facilitate for this, WMTS frequencies is used between the CCU and the base station allowing for a much larger range and operating in the 608-614 $\mathrm{MHz}$ band. This implies that the intermediate CCUs must be able to operate at both at MICS and WMTS frequencies to provide a link between the nodes and the computer. The CCU remains in close range with the patient and may be attached to their belt for example when it is for individual use. The nominal wireless distance for the MICS link is more 10 meters. The WMTS targets a distance more than $100 \mathrm{~m}$.

Two pieces of software were created in this project as indicated in Fig. 1. The software residing at the local PC is named GATEWAY. The job of GATEWAY is to gather data from the base station's CCU through RS232 cable and forwards it to the remote PC through Ethernet Sockets. The software residing at the Remote PC is named the BSN App. The BSN App will collate data from the local PC, interpret them and store them onto the remote $\mathrm{PC}$ to be analyzed later by health professionals. The receiver station (i.e. the remote PC) is capable of displaying all the received data on a User Display Graphic (GUI) and is also capable of storing all the data in the database system of a medical center (Section IV).

\section{SEnsor Nodes AND CCU Hardware Designs}

Sensor nodes are designed to be small and power efficient so that their battery last for a long time. They collect the weak raw signals from a human body. The most common physiological signals are EEG, ECG, Pulse Rate, Respiration Rate, Temperature, blood oxygen saturation $\left(\mathrm{SpO}_{2}\right)$, Blood Gas Level, Cardiac Output, Respiratory Gas and Blood Pressure. All these sensors are to be mounted onto the sensor nodes and their data are sent wirelessly to the CCU. Fig. 3 shows the hardware implementation of our sensor nodes. We built four sensor electronics (i.e. 4-channel) on a common PCB board so that some electronics can be used interchangeable. At this point we have temperature, pulse rate, ECG and another channel that is left free in order to be used for another important physiological signal in Future. The antennas for this project are designed as a loop printed around the prototyping boards (See Fig. 3).

We designed two different $\mathrm{CCU}$ boards to provide the scenario given in Fig. 1. One CCU is designed to connect a computer via the USB port (Fig. 4). The other CCU is used to function as an intermediate device (Fig. 5) that presents the WMTS wireless link. Although both CCUs can be used for multiple patients monitoring, the first CCU type (CCU-1) is more useful for private usage at home or in a room of a hospital for a single patient only.
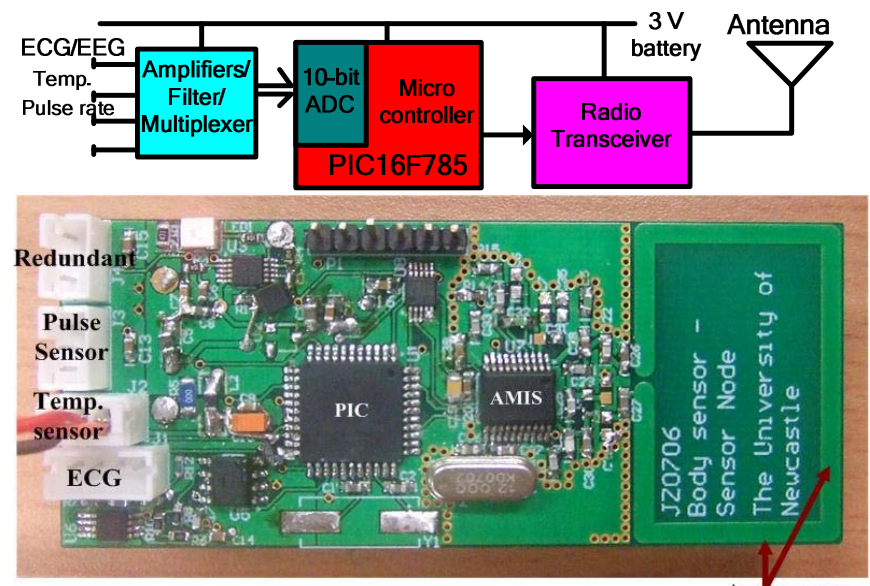

Antenna

Figure-3. A 4-channel sensor node hardware. 


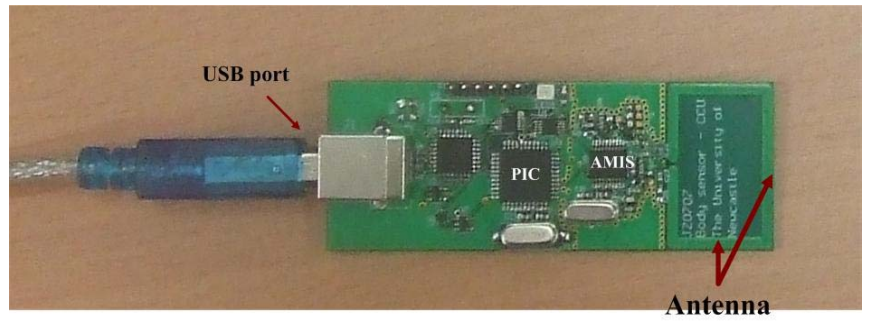

Figure-4. CCU-1: a central control unit (CCU) used individually.

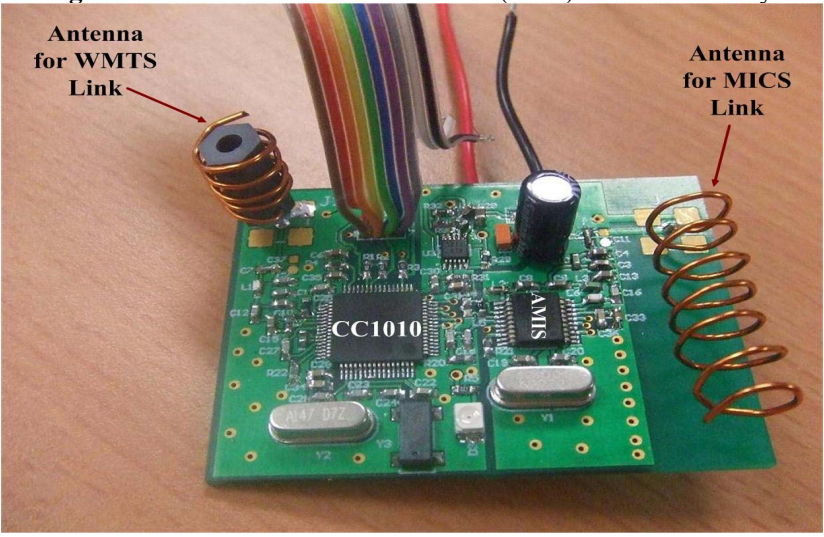

Figure-5. CCU-2: Intermediate Central Control Unit (CCU). This device is shared by more than one patient and portable. It receives signals from patients via the MICS wireless link and transmits to a base station via the WTMS wireless link. It uses two transceiver chips (AMIS and CC1010) to provide both wireless links.

The sensor nodes/CCU hardwares require a microcontroller and a wireless transceiver to coordinate all activities. The CCU-1/sensor nodes consist of the wireless link (AMI52100 IC) from AMI semiconductor and the microcontroller PIC16F87. In addition to these chips, we added another transceiver the CC1010 chip from Chipcon on the intermediate CCU (CCU-2) board to obtain a wireless transmission with the WMTS band. The antennas for both WMTS and MICS are made of coil. All the boards are custom made except the base station which is CC1010 evaluation module obtained from Chipcon. The CC1010 transceiver chip has the capability to transmit anywhere within 300 and $1000 \mathrm{MHz}$ (It was used for WMTS band). Fig 6 shows a spectrum plot of WMTS used during communication when FSK modulation mode is selected. The bandwidth of the signal is well within the bandwidth of the WMTS band $(608-615 \mathrm{MHz})$. In Future, the CCU-2 can include the standards such as Bluetooth, Zigbee, and the $802.11 \mathrm{Wi}-\mathrm{Fi}$ standards to accommodate different wireless platforms.

\section{Data Base, Software Programs and Monitoring}

As explained earlier, the multi-access communication protocol is a combination of CSMA/CA and polling architecture. Sensors to detect temperature, pulse rate and ECG are used to communicate with the base stations in the prototype system shown in Fig. 2. Pulse rate and temperature signals are low rate and can easily be transmitted [19]. The monitoring of the continuous signal like ECG is more complicated that gives rise to other continuous vital signals,

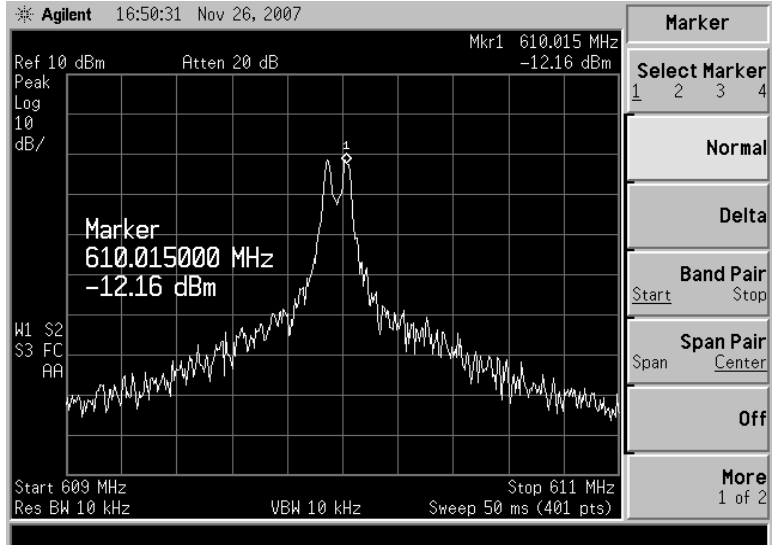

Figure 6. Spectrum for WMTS link

such as EEG and EMG monitoring as the signal amplification and filter circuits are very similar. Unlike temperature and pulse rate, the information sent to the $\mathrm{CCU}$ requires a continual and undisturbed sampling period as high as 400 samples per second, each sample of size 10 bits ( 4000 bps). To allow for MAC overhead and packet retransmission, the baud rate (i.e. data rate) for the RF link should be at least twice this rate. The use of Manchester encoding is advisable to help maintain data integrity and a stable connection, which halves the data rate of the link. Each sensor node representing only one patient can only have one ECG. Fig. 7 is an ECG signal obtained from our set up.

A database server has been developed to maintain data integrity which is necessary for big medical centers. A monitoring of ECG for an individual particular is shown in the Fig. 8. In GUI, a more detailed of patients' particulars can be seen from the database. Clicking on a patient shows the sensors attached to them and their personal information/picture. Clicking on a sensor displays the sensor's information (e.g. interval). It also brings up how many recordings are available. Single clicking a record shows when the record was made. Double clicking displays the record on the graph. In the following figure, it is arranged for an ECG monitoring. It's very handy to have a data file compatible with Matlab program for signal analysis as mentioned earlier.

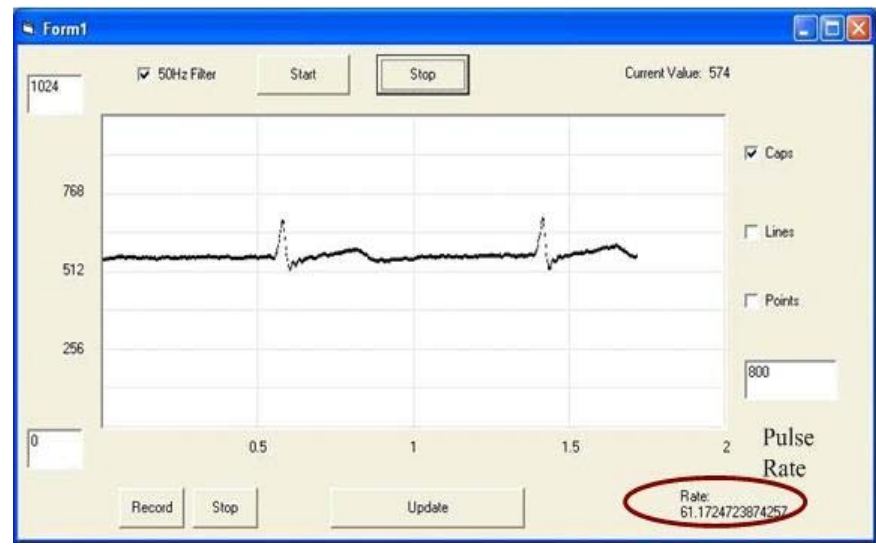

Figure-7 A clean wireless ECG monitoring showing pulse rate as well. 




Fi gure-8. Live monitoring of ECG via database.

\section{CONCLUSION}

In this paper we presented a multi hopping network for a WBAN system that can be used in medical environment for remote monitoring physiological parameters from many patients simultaneously. Issues related to a complete WBAN system to deploy in a medical center have been discussed. A prototype system that is able to acquire readings from multiple patients has been presented. After obtaining data from sensors, the data can be transferred to a remote PC through a local area network or the internet for further analysis. Such a wireless body sensor network system is very suitable to be used in hospitals environments to reduce human errors, to reduce health care cost, to provide more time to health professionals to deal with other important issues as well as to increase patients' comfort level as they no longer need to be wakening up for periodic checks. A discussion of wireless technologies has also been given in this paper.

We are working to interface our MICS/WMTS devices with other wireless standards such as WLANs, ZigBee (IEEE 802.15.4), mobile networks (e.g. GSM) or Bluetooth (IEEE 802.15.1) in order to extend the applications in different environments. Such a heterogonous wireless network can help to coordinate collaborations across medical centers.

\section{REFERENCES}

[1] FCC Rules and Regulations, "MICS Band Plan," Table of Frequency Allocations, Part 95, Jan. 2003.

[2] Australian Communications Authority, Radio Frequency Planning Group, "Planning for Medical Implant Communications Systems \& Related Devices", Proposals Paper, Oct. 2003.

[3] Wireless Medical Telemetry, FCC Part 952000.

[4] R. Fischer et al. SMART: Scalable Medical Alert and Response Technology. http://smart.csail.mit.edu/.

[5] T. Gao, D. Greenspan, M. Welsh, R. R. Juang and A. Alm, "vital signs monitoring and patient tracking over a wireless network," IEEE-EMBS 27th Annual Int. Conference of the Eng. in Medicine and Biology, Sept. 2005, Page(s): $102-105$.

[6] C. A. Otto, E. Jovanov, and E. Milenkovic, "A WBAN-based system for health monitoring at home," in 3rd IEEE/EMBS Int. Summer School, Medical Devices and Biosensors, Sept. 2006, Page(s):20 - 23.
[7] C. H. Chan, C. C. Y. Poon, Raymond C. S. Wong, and Y. T. Zhang, “ A hybrid body sensor network for continuous and long-term measurement of arterial blood pressure," 4th IEEE/EMBS International Summer School and Symposium on Medical Devices and Biosensors, 19-22 Aug. 2007, Page(s):121 - 123

[8] U. Anliker et al."AMON: a werable multiparameter medical monitoring and alert system, in IEEE Trans. Information Tech. In Biomedicine, vol. 8, Dec 2004.

[9] C. M. Jong, R. S. H. Istepanian, A. Alesanco, and H. Wang, "Hardware design \& compression issues in compact Bluetooth enabled wireless telecardiology system, IEEE 2nd International Conference on Broadband Networks, Oct. 2005, Page(s):1014 - 1015.

[10] M. F. A. Rasid and B. Woodward, "Bluetooth telemedicine Processor for multichannel biomedical signal transmission via mobile cellular networks," IEEE Transactions on Information Technology in Biomedicine, pp. 35- 43, Vol. 9, March 2005.

[11] N. F. Timmons, W. G. Scanlon, "Analysis of the performance of IEEE 802.15.4 for medical sensor body area networking", IEEE SECON 2004.

[12] N. Golmie, D. Cypher, and O. Rebala, "Performance analysis of low rate wireless technologies for medical applications" Computer Communications, vol. 28, pages 1266-1275, June 2005.

[13] S. Y. Shin, H. S. Park, and W. H. Kwon, "Mutual interference analysis of IEEE 802.15.4 and IEEE 802.11b," Computer Networks: The International Journal of Computer and Telecommunications Networking, Vol. 51 , August 2007, Pages 3338-3353.

[14] I. Howitt, and J. A Gutierrez, " IEEE 802.15.4 low rate - wireless personal area network coexistence issues," IEEE Wireless Commun. and Networking Conf. (WCNC), pp. 1481-1486, 2003.

[15] A Sikora and V. F. Groza "Coexistence of IEEE802. 15.4 with other Systems in the 2.4 GHz-ISM-Band", Proceedings of IEEE Instrumentation and Measurement, May 2005, pages 1776-1791.

[16] http://www.ieee802.org/15/pub/TG6.html, March 2008.

[17] L. Huan-Bang and R. Kohno, "Introduction of SG-BAN in IEEE 802.15 with related discussion," IEEE Intern. Conference Ultrawideband (ICUWB '07), Sept. 2007, Page(s):134 - 139.

[18] R. Gharpurey and P. Kinget, Ultra Wideband : Circuits, Transceivers and Systems. Springer US, 2008

[19] M. R. Yuce, P. C. Ng, C. K. Lee, J. Y. Khan, and W. Liu , "A wireless medical monitoring over a heterogeneous sensor network," in the 29th International Conference of the IEEE Engineering in Medicine and Biology Society, August, 2007, Page(s):5894 - 5898.

[20] Henry Higgins, "in-body RF communication and the future of Healthcare," Zarlink semiconductor.

[21] R. Diefes, Medical Telemetry Then and Now http://www.fda.gov/cdrh/medsun/AudioConf_files/ 